\title{
XXIV ASTIN COLLOQUIUM
}

The 24th ASTIN Colloquium will be held in the United Kingdom

The intention is to base the colloquium at St. John's College, Cambridge, for the period 25th-29th July 1993.

Further details and the preliminary registration form will appear in the IAA Bulletin, issue April 1992. 